DOI:http://dx.doi.org/10.18524/1810-4215.2019.32.182233

\title{
SPECTROSCOPIC INVESTIGATIONS OF GALACTIC CLUSTERS WITH ASSOCIATED CEPHEID VARIABLES. III. COLLINDER 394 AND BB SGR
}

\author{
I. A. Usenko ${ }^{1,2}$, A. Yu. Kniazev ${ }^{3,4}$, I. Yu. Katkov ${ }^{5}$, V. V. Kovtyukh ${ }^{1}$, \\ T. V. Mishenina ${ }^{1}$, A. S. Miroshnichenko ${ }^{6,7,8}$, D. G. Turner ${ }^{9}$ \\ 1 Astronomical Observatory, Odessa National University, Shevchenko Park, \\ Odessa 65014, Ukraine, vkovtyukh@ukr.net \\ 2 Mykolaiv Astronomical Observatory, Obsevatorna 1, Mykolaiv 54030, Ukraine, \\ igus99@ukr.net \\ 3 South African Astronomical Observatory, P.O. 7925, Cape Town, \\ South Africa, akniazev@saao.ac.za \\ ${ }^{4}$ Southern African Large Telescope Foundation, P.O. 7925, Cape Town, \\ South Africa \\ ${ }^{5}$ New York University, Abu Dhabi, Saadiyat Island, P.O. 129188 \\ Abu Dhabi, UAE, ik52@nyu.edu \\ ${ }^{6}$ Dept. of Physics and Astronomy, University of North Carolina at \\ Greensboro, P.O.Box 261170, Greensboro, NC 27402, USA, a_mirosh@uncg.edu \\ ${ }^{7}$ Main Astronomical Observatory of the Russian Academy of Sciences, \\ Pulkovskoe shosse 65-1, Saint-Petersburg, 196140, Russia \\ ${ }^{8}$ Fesenkov Astrophysical Institute, Observatory 23, Almaty, 50020, Kazakhstan \\ ${ }^{9}$ Dept. of Astronomy and Physics, Saint Mary's University, \\ 923 Robie Street, Halifax B3H3C3, Nova Scotia, Canada, turner@ap.smu.ca
}

\begin{abstract}
We present the results of a spectroscopic and photometric investigation of 20 objects from the open cluster Collinder 394, which contains the Cepheid BB Sgr. Besides the Cepheid, we studied tree K-giants, four B-giants, and twelve B-A-F main sequence stars. Radial velocities $(\mathrm{RV}), \mathrm{v} \sin \mathrm{i}, T_{\text {eff }}$, $\log \mathrm{g}$, were determined using spectroscopic model fitting and atmosphere models. We have derived the color-excesses, reddenings, and intrinsic colors for these stars using their $T_{\text {eff }}$ and $\log g$ from comparison to the atmosphere models, especially for hot stars. Proper motions, RV and GAIA DR2 2018 parallax/distance values for these stars allowed us to determine their membership in the cluster and absolute magnitudes. We found that seven stars do not belong to the cluster. The parallaxes and reddenings of 13 confidently cluster members led to the distances in a range of $630-800 \mathrm{pc}$, although the majority of them $(8$ objects $)$ are located at a mean distance of $657.7 \pm 66.7 \mathrm{pc}$, and 5 objects with the Cepheid could probably belong to the cluster's corona. All the members have $[\mathrm{Fe} / \mathrm{H}]$ near 0.1 dex. The main sequence B-star No. 76 (HD 174307) has a
\end{abstract}

low rotational velocity projection, and this fact allows us to determine its chemical composition to compare with that of the Cepheid BB Sgr. Its CNO and $\mathrm{Na}$ abundances are close to the solar one, while the Cepheid show a deficit of carbon, an overabundance of nitrogen, nearly solar oxygen, and an overabundance of sodium. Three foreground K-giants have different $\mathrm{C}$ abundances (from a deficit to the solar), and $\mathrm{Na}$ (from the solar to an overabundance) as well as a solar-like O content. The foreground F7-8 V star No. 75 (CPD $\left.-20^{\circ} 7218\right)$ was revealed to be a Li-rich star, while the A7 V star No. 69 (BD -20 5290$)$ has an anomalous deficit of $\mathrm{CNO}$ with overabundances of some $\alpha, \mathrm{r}$ - and s-process elements.

Key words: Open clusters: radial velocities; Stars: abundance; GAIA parallaxes; Cepheids; B - giants; K-giants; individual: Collinder 394

АНОТАЦІЯ. Ми презентуємо результати спектроскопічних та фотометрічних дослідів 20 об'єктів з розсіяного скупчення Collinder 394, яке вміщує цефеїду BB Sgr. Окрім цефеїди, ми 
дослідили три К-гіганта, чотири В-гіганта та дванадцать В-A-F зір. Радіальні швидкості (RV), $\mathrm{v} \sin \mathrm{i}, T_{\mathrm{eff}}, \log$ gотримані з використанням методів спектроскопічного фіттінгу та моделей атмосфер. Ми вивели надлишки кольорів, почервоніння та справжні кольори ціх зір, використовуючи їх $T_{\text {eff та }}$ $\log$ gшляхом порівнювання $з$ моделями атмосфер, особливо для горячих зір. Власні рухи, RV та паралакси/відстані з GAIA DR2 2018 для ціх зір дозволили встановити їх членство у скупченні та абсолютні магнітуди. Встановлено, що сім зір не належать до скупчення. Паралакси та почервоніння 13 справжніх членів скупчення дають відстані від 630 до 800 пс, хоча більшисть з них (8 об'єктів) знаходяться на середньої відстані у $657.7 \pm 66.7$, та п'ять з цефеїдою можливо, належать до корони скупчення. Усі члени мають $[\mathrm{Fe} / \mathrm{H}]$ біля 0.1 dex. В-зоря ГП No. 76 (HD 174307) має мале значення проєкції швидкості оберту, та це дозволило встановити їі хімічний склад для порівняння з BB Sgr. Ï̈ вміст CNO та Na близький до сонячного, тоді як цефеїда має дефіціт вуглецю, надлишок азоту, сонячний кисень та надлишок натрію. Три К-гіганти перед скупченням мають різний вміст вуглецю (від дефіціту до сонячного) та натрію (від сончного до надлишку), та сонячний кисень. Зоря No. 75 (CPD - 207218) (F7-8 V), яка теж є перед скупченням, виявилась з надлишком $\mathrm{Li}$, тоді як зоря No. 69 (BD -205290) (A7 V) має аномальний дефіціт $\mathrm{CNO} 3$ надлишком деяких елементів $\alpha, \mathrm{r}$ - та s- процесів.

Ключові слова: Розсіяні скупчення, променеві швидкості, хімічний склад зір, паралакси GAIA, цефеїди, В-гіганти, К-гіганти, скупчення Collinder 394.

\section{Introduction}

The open cluster Collinder 394 is part of a double cluster with Collinder 393 (NGC 6716) (Turner \& Prederos 1985). Being a loose grouping of $B$ - and $A$ type stars, it contains a $6 .^{d} 64$ Cepheid BB Sgr as a possible member of its corona. According to Turner $\&$ Prederos (1985), the earliest spectral type stars in it are $\left.\mathrm{B} 8, E_{(} B-V\right)=0.25 \pm 0.01, \mathrm{~V}_{0}-\mathrm{M}_{V}=9.04 \pm 0.08, d$ $=643 \pm 25 \mathrm{pc}$, and an age near $6 \times 10^{7} \mathrm{yr}$. Collinder 394 has not been carefully studied spectroscopically except for BB Sgr. Therefore, the main goals of our investigation are as follows: 1) to measure the radial and rotational velocities of the cluster's stars, 2) to determine the atmospheric parameters, metallicities, CNO and $\mathrm{Na}$ abundances and compare the abundances of the objects of different spectral types, and 3) to determine the distances using GAIA DR2 parallaxes and RV data, and to check thier membership in the cluster.

\section{Observations}

Our observations were taken at the $11 \mathrm{~m}$ SALT (Southern African Large Telescope) equipped with HRS (High Resolution Spectrograph). HRS is a dualbeam (3700-5500 \& 5500-8900 ̊) fiber-fed, whitepupil, échelle spectrograph, which uses VHP gratings as cross dispersers. We obtained one spectrum for each object using the medium mode with the spectral resolving power $R=40000$, an average $\mathrm{S} / \mathrm{N}$ of over 100 , which it is enough to reach our observational goals. These spectra will be used to derive the atmosphere parameters and chemical abundances for some elements of the open cluster members. The data were reduced using the échelle context in MIDAS. Also, the feros package developed for échelle data reduction from the Fiber-fed Extended Range Optical Spectrograph (FEROS) was used. Both FEROS and HRS provide very similar échelle data.

We used the DECH30 package (Galazutdinov 2007) designed to use the spectra in FITS format to measure the line depths and their equivalent widths. The radial and rotational velocities were measured by fitting of the observed spectra with models from Coelho (2014). The object IDs, magnitudes, spectral types, proper motions, parallaxes from the GAIA DR18 catalogue, measured radial and rotational velocities for each spectrum are given in Table 1.

\section{Results and Analysis}

3.1. Radial and rotational velocities, proper motions and parallaxes

As seen in Table 1, judging from proper motion, radial velocity and parallax measurements, seven objects are evident foreground or background stars. It should be noted that our results are consistent with those from other sources: $\mathrm{RV}=12.0 \mathrm{~km} \mathrm{~s}^{-1}$ (Wilson \& Joy 1950) for $\mathrm{HD} 124403$; and $\langle\mathrm{RV}\rangle=11.35 \mathrm{~km} \mathrm{~s}^{-1}$ (LloydEvans 1968) for BB Sgr, respectively. According to Conrad et al. (2017), the cluster's mean radial velocity is $-1.9 \pm 6.5 \mathrm{~km} \mathrm{~s}^{-1}$, and the majority of confident cluster members have close radial velocities.

As seen in Table 1, the Cepheid BB Sgr, three Kgiants Nos. 21, 72, 74 and the F7 V star No. 75 have low vini, while hot main-sequence (hereafter MS) stars are fast-rotating objects. It is very surprisingly to detect the MS B-star No. 76 with a low radial velocity projection.

3.2. Color-indices, color-excesses, reddenings, and atmosphic parameters

Turner \& Prederos (1985) determined color-excesses, reddenings, and intrinsic colors for the cluster's ob- 
jects using $U B V R I$ color-indices. To improve these parameters, we have used their atmospheric parameters $T_{\text {eff }}$ and $\log g$, determined with the model fitting method. Next, these estimates were performed for the $(\mathrm{U}-\mathrm{B})_{0}$ and $(\mathrm{B}-\mathrm{V})_{0}$ determinations for the hot Band A-type stars using the Kurucz (1993) models from Bessel et al. (1998). For more evolved stars of $F G K$ spectral types with a large number of narrow metallic absorption lines we have used the lines depth relation to derive $T_{\text {eff }}$ (Kovtyukh 2007), and a Fe ionization balance to derive $\log g$. These estimates give an opportunity to determine corresponding $(\mathrm{U}-\mathrm{B})_{0}$ and $(\mathrm{B}-\mathrm{V})_{0}$ values for $F G K$-stars.

The described above results allowed us to determine the color-excesses, reddenings, and interstellar extinction. All these data are given in Table 2. At that we have used (U-B) and (B-V) color-indices from Turner \& Prederos (1985). As seen from this table, the majority of hot stars have close reddening values in a range of $0 .^{m} 56-0 .^{m} 88$, except for the Be star No. 24 (HD 174652) and stars, which probably belong to the cluster's corona: HD 174403, Nos. 70 and 76, and BB Sgr $\left(0 .^{m} 97-1 .^{m} 19\right)$. Our $\mathrm{E}_{B-V}$ values demonstrate an excellent agreement with photometrically determined ones from Turner \& Prederos (1985) Table 1, except for the star No. 30. The foreground and background objects have very different parameters. Figure 1 represents a $T_{\text {eff }}-\mathrm{M}_{V}$ diagram for the confident members of Collinder 394. As seen from the diagram, object No. 76 with a low rotation velocity projection is located in the middle of the cluster's MS, while the object No. 61 is located near the "turn-off" point. HD 174403, an eclipsing binary (Turner \& Prederos 1983), was found at the horizontal branch. Its main component with a mass of $6 \mathrm{M}_{\odot}$ could be a first-crossing Cepheid instability strip object.

\subsection{Absolute magnitudes and distances}

Table 3 contains the absolute magnitudes of the Collinder 394 objects derived from photometry by Turner \& Prederos (1985) and those derived by us using the GAIA DR2 parallaxes and our $T_{\text {eff }}$ and $A_{V}$ data. As can be seen from the Table 3, the parallaxbased $\mathrm{M}_{V}$ only for 8 objects determined as the confident cluster's members agree with those from Turner \& Prederos (1985). Figure 2 represents a variableextinction diagram for the cluster confident members only. We have obtained the mean distance modulus $\left(\mathrm{V}_{0}-\mathrm{M}_{V}\right)=9.09 \pm 0.21$ with the reddening law slope $\mathrm{R}$ $=\mathrm{A}_{V} / \mathrm{E}_{B-V}=3.34 \pm 0.02$. Turner \& Prederos (1985) estimated $\mathrm{R}=3.1 \pm 0.3$. Our estimate of $\left(\mathrm{V}_{0}-\mathrm{M}_{V}\right)$ gives a distance of $\mathrm{d}=657.7 \pm 66.7 \mathrm{pc}$, and with this value it turns out that Collinder 394 is located about 15 pc further away compared to the value from Turner $\&$ Prederos (1985). Four objects (Nos. 12, 27, 63, and 76) located at 760-824 pc could belong to the corona, similar to BB Sgr, which has $\mathrm{M}_{V}=-3 .^{m} 59 \pm 0.07$ and $\mathrm{d}=801 \pm 26.4 \mathrm{pc}$, respectively.

Other objects from the list turned out to be either foreground or background stars - all three K-giants, and three MS A- and F- type stars.

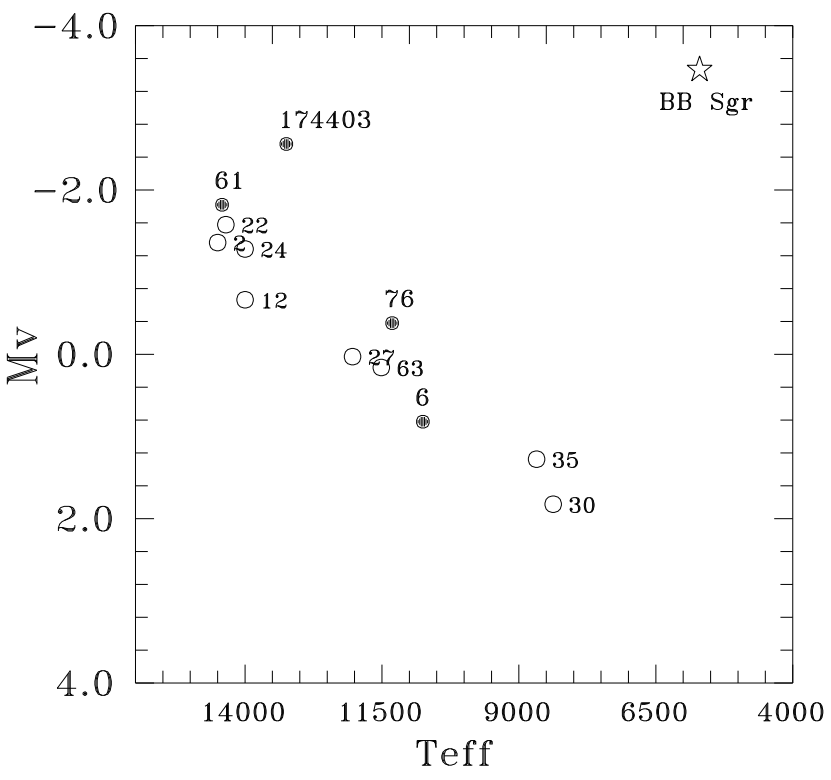

Figure 1: $T_{\text {eff- }} \mathrm{M}_{V}$ diagram for the Collinder 394 confident members. Open circles show the MS stars, filled circles show the B-giants.

\subsection{Chemical abundances}

When the atmospheric parameters were derived, we used the VALD oscillator strengths (Kupka et al. 1999) and LTE model atmospheres from Castelli \& Kurucz (2004) for determination of the element abundances. For that we have worked with stars with relatively low rotational velocity projections: BB Sgr, Nos. 21, 69, $72,74,75$, and 76 . As seen, only two of these objects are true cluster members, therefore the rest are foreground and background ones, respectively. Nevertheless the chemical abundances of each object from our list are of interest. Tables 4 and 5 present the results of our investigations. Since these objects have different evolutionary stages, we will discuss every star separately.

BB Sgr The Cepheid has a solar-like iron content, a carbon deficit, an overabundance of nitrogen and sodium, and a nearly solar oxygen abundance, i.e. typical for a yellow supergiant after the first dredge-up stage. All other elements have nearly solar abundances. Therefore, our results are close to those of Usenko et al. (2013) and Luck et al. (2006).

76 (HD 174307) This MS B-star, which is considered to be a Cepheid progenitor, demonstrates a solar CNO, Na, Fe, and other elements abundances with small overabundances of neon, argon, and sulphur, 
Table 1: General data for the observed objects in Collinder 394 and derived RVs.

\begin{tabular}{|c|c|c|c|c|c|c|c|}
\hline Object & $V$ & Sp.type & $\mu_{\alpha}$ & $\mu_{\delta}$ & $\mathrm{RV}, \mathrm{km} \mathrm{s}^{-1}$ & PLX (GAIA) & Membership \\
\hline BB Sgr ${ }^{1}$ & 6.93 & G0 Ib & +0.392 & -5.078 & $+17.11 \pm 0.04$ & $1.2485 \pm 0.0411$ & $\mathrm{~m}$ \\
\hline HD 174403 & 7.51 & B8 II-III & -1.477 & -5.987 & $+18.76 \pm 0.43$ & $1.5011 \pm 0.0529$ & $\mathrm{~m}$ \\
\hline $2(\mathrm{HD} 174723)$ & 8.88 & B8 & -1.468 & -5.838 & $+9.51 \pm 0.23$ & $1.3703 \pm 0.0622$ & $\mathrm{~m}$ \\
\hline $3\left(\mathrm{CPD}-20^{\circ} 7272\right)$ & 11.19 & B9? & -65.800 & +6.600 & $+30.24 \pm 0.12$ & & $\mathrm{~nm}$ \\
\hline $6(\mathrm{HD} 174706)$ & 10.30 & B7-B8 II & -1.329 & -5.268 & $+3.57 \pm 0.24$ & $1.5783 \pm 0.0708$ & $\mathrm{~m}$ \\
\hline $12(\mathrm{HD} 174685)$ & 9.52 & $\mathrm{~B} 8 \mathrm{~V}$ & -1.967 & -6.206 & $+9.35 \pm 0.25$ & $1.2743 \pm 0.0716$ & $\mathrm{~m}$ \\
\hline $21\left(\mathrm{CPD}-20^{\circ} 7255\right)$ & 10.45 & KO III? & +1.662 & -12.445 & $-2.72 \pm 0.02$ & $2.2489 \pm 0.0673$ & fg \\
\hline $22(\mathrm{HD} 174651)$ & 8.47 & B8 IV & -2.256 & -6.227 & $+5.76 \pm 0.19$ & $1.4318 \pm 0.0777$ & $\mathrm{~m}$ \\
\hline $24(\mathrm{HD} 174652)$ & 9.06 & B9e & -1.417 & -6.317 & $-0.78 \pm 1.10$ & $1.3518 \pm 0.0612$ & $\mathrm{~m}$ \\
\hline $27\left(\mathrm{CPD}-20^{\circ} 5300\right)$ & 10.34 & B7-B8? & -1.310 & -5.728 & $+4.84 \pm 0.24$ & $1.2135 \pm 0.0546$ & $\mathrm{~m}$ \\
\hline $30\left(\mathrm{CPD}-20^{\circ} 7248\right)$ & 11.56 & AV & -2.052 & -5.579 & $-4.40 \pm 0.09$ & $1.5077 \pm 0.0487$ & $\mathrm{~m}$ \\
\hline $35\left(\mathrm{CPD}-20^{\circ} 7240\right)$ & 11.25 & $\mathrm{AV}$ & -0.909 & -5.493 & $+11.45 \pm 0.31$ & $1.3115 \pm 0.0473$ & $\mathrm{~m}$ \\
\hline 61 (HD 174594) & 8.30 & B5 III & -1.732 & -6.284 & $+8.77 \pm 0.18$ & $1.4439 \pm 0.0578$ & $\mathrm{~m}$ \\
\hline 63 (HD 174538) & 10.37 & B9 III & -4.300 & -6.800 & $+10.99 \pm 0.13$ & $1.2839 \pm 0.0503$ & $\mathrm{~m}$ \\
\hline $69\left(\mathrm{BD}-20^{\circ} 5290\right)$ & 10.87 & A7 V? & -0.115 & -9.890 & $-44.24 \pm 0.04$ & $2.4470 \pm 0.0439$ & fg \\
\hline 70 (GSC 06289-03051) & 12.25 & A2 $\mathrm{V}$ & -1.772 & -4.033 & $+27.50 \pm 0.32$ & $0.8821 \pm 0.0373$ & $\mathrm{bg}$ \\
\hline 72 (GSC 06289-03067) & 11.59 & K1 III? & -4.921 & -11.509 & $-46.78 \pm 0.04$ & $1.0858 \pm 0.0457$ & fg \\
\hline 74 (HD 174402) & 9.27 & $\mathrm{~K} 1 / 2$ III? & -25.509 & -43.853 & $-7.61 \pm 0.02$ & $3.7919 \pm 0.0453$ & 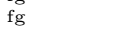 \\
\hline $75\left(\mathrm{CPD}-20^{\circ} 7218\right)$ & 11.11 & F7-8 V? & +9.694 & -4.597 & $-22.94 \pm 0.04$ & $4.7670 \pm 0.0357$ & fg \\
\hline 76 (HD 174307) & 10.36 & B8/9 II & -1.289 & -6.078 & $+17.00 \pm 0.05$ & $1.3155 \pm 0.0349$ & $\mathrm{~m}$ \\
\hline
\end{tabular}

1 - Phase $0 .{ }^{P} 624$ according to Usenko et al. (2013); $\mathrm{m}$ - member; nm - non-member; fg - foreground object; bg - background object.

Table 2: Atmospheric parameters, rotational velocities, intrinsic colors, color-excesses, reddenings, and extinctions for the Collinder 394 objects.

\begin{tabular}{|c|c|c|c|c|c|c|c|c|c|}
\hline Star & $T_{\text {eff }}$ & $\log g$ & $\mathrm{v} \sin \mathrm{i}$ & $(\mathrm{U}-\mathrm{B})_{0}$ & $(\mathrm{~B}-\mathrm{V})_{0}$ & $\mathrm{E}_{U-B}$ & $\mathrm{E}_{B-V}$ & $\mathrm{~A}_{V}$ & $R$ \\
\hline $\mathrm{BB} \mathrm{Sgr}^{\mathrm{I}}$ & $5332 \pm 6$ & $1.30 \pm 0.05$ & $10.8 \pm 0.1$ & 0.61 & 0.87 & 0.30 & 0.28 & 1.00 & 3.57 \\
\hline HD 174403 & $13245 \pm 165$ & $3.49 \pm 0.01$ & $198.7 \pm 1.4$ & -0.48 & -0.13 & 0.22 & 0.29 & 0.97 & 3.33 \\
\hline $2(\mathrm{HD} 174723)$ & $14500 \pm 10$ & $4.00 \pm 0.01$ & $137.9 \pm 0.4$ & -0.54 & -0.14 & 0.15 & 0.25 & 0.84 & 3.32 \\
\hline $3\left(\mathrm{CPD}-20^{\circ} 7272\right)$ & $10700 \pm 7$ & $4.42 \pm 0.02$ & $110.5 \pm 0.3$ & -0.16 & -0.04 & 0.25 & 0.26 & 0.88 & 3.35 \\
\hline $6(\mathrm{HD} 174706)$ & $10752 \pm 10$ & $4.32 \pm 0.02$ & $132.8 \pm 1.3$ & -0.17 & -0.05 & 0.20 & 0.26 & 0.87 & 3.35 \\
\hline 12 (HD 174685) & $14000 \pm 50$ & $4.30 \pm 0.01$ & $158.1 \pm 0.6$ & -0.49 & -0.13 & 0.17 & 0.23 & 0.76 & 3.32 \\
\hline $21\left(\mathrm{CPD}-20^{\circ} 7255\right)$ & $4825 \pm 13$ & $3.00 \pm 0.02$ & $0.0 \pm 0.0$ & 0.78 & 1.03 & 0.10 & 0.13 & 0.47 & 3.65 \\
\hline $22($ HD 174651) & $14350 \pm 77$ & $4.00 \pm 0.01$ & $223.3 \pm 0.6$ & -0.53 & -0.14 & 0.22 & 0.25 & 0.84 & 3.32 \\
\hline $24(\mathrm{HD} 174652)$ & $14000 \pm 200$ & $3.62 \pm 0.50$ & $237.3 \pm 20.3$ & -0.53 & -0.14 & 0.23 & 0.30 & 1.01 & 3.35 \\
\hline $27\left(\mathrm{CPD}-20^{\circ} 5300\right)$ & $12037 \pm 12$ & $4.19 \pm 0.01$ & $129.7 \pm 0.5$ & -0.32 & -0.10 & 0.15 & 0.22 & 0.74 & $\begin{array}{l}3.00 \\
3.36\end{array}$ \\
\hline $30\left(\mathrm{CPD}-20^{\circ} 7248\right)$ & $8375 \pm 2$ & $4.17 \pm 0.01$ & $59.1 \pm 0.3$ & 0.08 & 0.13 & 0.12 & 0.19 & 0.63 & 3.36 \\
\hline $35\left(\mathrm{CPD}-20^{\circ} 7240\right)$ & $8676 \pm 7$ & $3.63 \pm 0.01$ & $314.9 \pm 1.0$ & 0.10 & 0.05 & 0.07 & 0.17 & 0.56 & 3.37 \\
\hline 61 (HD 174594) & $14418 \pm 19$ & $\begin{array}{l}3.03 \pm 0.01 \\
3.78 \pm 0.01\end{array}$ & $155.7 \pm 0.6$ & -0.55 & $\begin{array}{l}0.05 \\
-0.15\end{array}$ & 0.17 & 0.26 & 0.84 & $\begin{array}{l}3.01 \\
3.29\end{array}$ \\
\hline 63 (HD 174538) & $11511 \pm 6$ & $4.17 \pm 0.01$ & $229.2 \pm 0.6$ & -0.26 & -0.08 & 0.18 & 0.26 & 0.88 & 3.35 \\
\hline $69\left(\mathrm{BD}-20^{\circ} 5290\right)$ & $7705 \pm 5$ & $3.50 \pm 0.01$ & $1.9 \pm 0.2$ & 0.19 & 0.16 & 0.31 & 0.34 & 1.17 & $\begin{array}{l}3.40 \\
3.40\end{array}$ \\
\hline 70 (GSC 06289-03051) & $9698 \pm 15$ & $3.81 \pm 0.02$ & $272.4 \pm 1.3$ & -0.40 & -0.02 & 0.65 & 0.35 & 1.19 & 3.36 \\
\hline 72 (GSC 06289-03067) & $4683 \pm 12$ & $2.70 \pm 0.02$ & $0.1 \pm 0.0$ & 0.93 & 1.10 & 0.38 & 0.27 & 0.99 & 3.68 \\
\hline 74 (HD 174402) & $4575 \pm 19$ & $3.10 \pm 0.02$ & $7.9 \pm 0.1$ & 0.98 & 1.12 & 0.11 & 0.13 & 0.48 & $\begin{array}{l}3.00 \\
3.68\end{array}$ \\
\hline $75\left(\mathrm{CPD}-20^{\circ} 7218\right)$ & $6250 \pm 15$ & $4.50 \pm 0.05$ & $24.7 \pm 0.1$ & 0.01 & 0.54 & 0.04 & 0.05 & 0.19 & 3.42 \\
\hline 76 (HD 174307) & $11313 \pm 13$ & $3.87 \pm 0.01$ & $0.5 \pm 0.1$ & -0.25 & -0.09 & 0.19 & 0.35 & 1.17 & 3.35 \\
\hline
\end{tabular}

$1-T_{\text {eff }}, \log \mathrm{g},(\mathrm{U}-\mathrm{B})_{0}$ and $(\mathrm{B}-\mathrm{V})_{0}$ for the phase 0.624 according to Usenko et al. (2013)

Table 3: Comparison between absolute magnitudes and distances, determined using GAIA DR2 parallaxes and photometric data.

\begin{tabular}{|c|c|c|c|}
\hline Object & $\mathrm{M}_{V}(\mathrm{~T} \& \mathrm{P} 85)$ & $\mathrm{M}_{V}$ (GAIA) & $\mathrm{d}(\mathrm{pc})(\mathrm{GAIA})$ \\
\hline BB Sgr & $-3.08 \pm 0.12$ & $-3.59 \pm 0.07$ & $800.96 \pm 26.37$ \\
\hline HD 174403 & $-2.44 \pm 0.08$ & $-2.56 \pm 0.00$ & $666.18 \pm 23.48$ \\
\hline $2(\mathrm{HD} 174723)$ & $-1.03 \pm 0.05$ & $-1.36 \pm 0.10$ & $729.77 \pm 33.13$ \\
\hline $3\left(\mathrm{CPD}-20^{\circ} 7272\right)$ & $+1.36 \pm 0.08$ & $+2.07 \pm 0.06$ & $444.66 \pm 13.31$ \\
\hline $6(\mathrm{HD} 174706)$ & $+0.44 \pm 0.08$ & $+0.42 \pm 0.10$ & $633.59 \pm 28.42$ \\
\hline 12 (HD 174685) & $-0.27 \pm 0.07$ & $-0.67 \pm 0.12$ & $784.75 \pm 44.09$ \\
\hline $21\left(\mathrm{CPD}-20^{\circ} 7255\right)$ & & $+1.74 \pm 0.06$ & $444.66 \pm 13.31$ \\
\hline $22(\mathrm{HD} 174651)$ & $-1.35 \pm 0.08$ & $-1.58 \pm 0.11$ & $698.42 \pm 37.90$ \\
\hline $24(\mathrm{HD} 174652)$ & $-0.95 \pm 0.08$ & $-1.28 \pm 0.10$ & $739.75 \pm 33.49$ \\
\hline $27\left(\mathrm{CPD}-20^{\circ} 5300\right)$ & $+0.62 \pm 0.08$ & $+0.02 \pm 0.10$ & $824.06 \pm 37.08$ \\
\hline $30\left(\mathrm{CPD}-20^{\circ} 7248\right)$ & $+1.48 \pm 0.08$ & $+1.82 \pm 0.07$ & $663.26 \pm 21.42$ \\
\hline $35\left(\mathrm{CPD}-20^{\circ} 7240\right)$ & $+1.58 \pm 0.08$ & $+1.58 \pm 0.08$ & $762.49 \pm 27.50$ \\
\hline 61 (HD 174594) & $-1.68 \pm 0.08$ & $-1.82 \pm 0.09$ & $692.57 \pm 27.72$ \\
\hline 63 (HD 174538) & $+0.51 \pm 0.08$ & $+0.16 \pm 0.08$ & $778.88 \pm 30.51$ \\
\hline $69(\mathrm{BD}-20 \quad 5290)$ & $+0.83 \pm 0.08$ & $+1.64 \pm 0.04$ & $408.66 \pm 7.33$ \\
\hline 70 (GSC 06289-03051) & $+2.23 \pm 0.08$ & $+0.79 \pm 0.09$ & $1133.66 \pm 47.94$ \\
\hline $72($ GSC $06289-03067)$ & & $+0.78 \pm 0.09$ & $920.98 \pm 38.76$ \\
\hline $74(\mathrm{HD} 174402)$ & - & $+1.68 \pm 0.02$ & $263.72 \pm 3.15$ \\
\hline $75\left(\mathrm{CPD}-20^{\circ} 7218\right)$ & & $+4.31 \pm 0.01$ & $209.78 \pm 1.57$ \\
\hline 76 (HD 174307) & $+0.22 \pm 0.08$ & $-0.38 \pm 0.05$ & $760.17 \pm 20.17$ \\
\hline
\end{tabular}

and a deficit of titanium.

69 (BD -205290) A MS A-star with a unique substantial CNO deficit, a deficit of $\mathrm{Mg}, \mathrm{Al}, \mathrm{Si}, \mathrm{Ca}$, $\mathrm{Sc}, \mathrm{Ti}$, a solar-like Fe, and an overabundance of $\mathrm{r}-$ and s-process elements.

$21\left(C P D-20^{\circ} 7255\right)$ An early K-giant, whith an upper estimate of lithium content $\log \mathrm{A}(\mathrm{Li})<0.4$ dex. It has a deficit of carbon, a small deficit of nitrogen and a close to solar abundance of oxygen and sodium. The rest of the elements show close to solar abundances except for a small overabundance of some s-process elements, an evident overabundance of $\mathrm{K}$ and $\mathrm{Mn}$, and a small deficit of $\mathrm{Rb}$.

72 (GSC 06289-03067) A colder K-giant has close to solar $\mathrm{C}$ and $\mathrm{O}$ abundances, a small overabundance of sodium and aluminum, the rest of the elements have a close to solar abundances, except for small overabundances of Si, S, Mn, and Eu.

74 (HD 174402) K-giant, that has solar abundances of $\mathrm{C}, \mathrm{O}$, and $\mathrm{Na}$. The rest of the elements have abundances close to solar one, except for small overabundances of S, Sc, Sm, and Eu.

75 (CPD - 20 $\left.0^{\circ} 7218\right)$ This F-type MS star is a lithiumrich object (see Fig. 3) with $\log \mathrm{A}(\mathrm{Li})=2.95$. CNO and $\mathrm{Na}$ abundances are close to solar, but some elements, such as K, V, Zr, Pr, Sm, Eu and Gd, demonstrate evident overabundances. 
Table 4: Chemical abundances for Collinder 394 objects. Part 1.

\begin{tabular}{|c|c|c|c|c|c|c|c|c|c|c|c|}
\hline & \multicolumn{5}{|c|}{ BB Sgr } & \multicolumn{3}{|c|}{76 (HD 174307) } & \multicolumn{3}{|c|}{$69\left(\mathrm{BD}-20^{\circ} 5290\right)$} \\
\hline Element & {$[\mathrm{El} / \mathrm{H}]$} & $\sigma$ & NL & UAL13 & LKA06 & {$[\mathrm{El} / \mathrm{H}]$} & $\sigma$ & NL & {$[\mathrm{El} / \mathrm{H}]$} & $\sigma$ & NL \\
\hline $\begin{array}{l}\text { C I } \\
\text { C } \text { II }\end{array}$ & -0.22 & 0.21 & 13 & -0.21 & -0.06 & 0.07 & 0.13 & 4 & -1.18 & 0.13 & 9 \\
\hline $\mathrm{N} \mathrm{I}$ & 0.46 & 0.24 & 4 & - & - & 0.05 & 0.14 & 9 & -1.45 & 0.18 & 6 \\
\hline O I & 0.03 & 0.02 & 2 & 0.02 & -0.13 & -0.07 & 0.08 & 7 & -0.92 & 0.12 & 3 \\
\hline $\mathrm{Ne} I$ & & & & & & 0.23 & 0.12 & 5 & & & \\
\hline $\mathrm{Na} I$ & 0.26 & 0.10 & 2 & & & -0.04 & 0.04 & 2 & -0.02 & 0.19 & 3 \\
\hline $\begin{array}{l}\mathrm{Mg} \mathrm{I} \\
\mathrm{Mg}_{\mathrm{II}}\end{array}$ & 0.01 & 0.00 & 1 & 0.26 & - & $\begin{array}{l}-0.02 \\
-0.05\end{array}$ & $\begin{array}{l}0.12 \\
0.15\end{array}$ & $\begin{array}{l}3 \\
7\end{array}$ & -0.51 & 0.04 & 2 \\
\hline Al I & 0.25 & 0.12 & 5 & 0.45 & 0.15 & & & & -0.24 & 0.15 & 5 \\
\hline Al II & & & & & & 0.07 & 0.14 & 5 & & & \\
\hline Si I & 0.17 & 0.11 & 16 & 0.25 & 0.14 & & & & -0.24 & 0.18 & 14 \\
\hline Si II & 0.18 & 0.33 & 2 & 0.21 & & -0.02 & 0.15 & 15 & 0.18 & 0.21 & 2 \\
\hline $\begin{array}{l}\text { S I } \\
\text { C I }\end{array}$ & 0.12 & 0.21 & 2 & -0.05 & -0.17 & & & & -0.13 & 0.21 & 3 \\
\hline $\begin{array}{l}\text { K II } \\
\text { I } 11\end{array}$ & & & & & & 0.25 & 0.17 & 15 & -0.04 & 0.00 & 1 \\
\hline Ar I & & & & & & 0.33 & 0.03 & 3 & & & \\
\hline $\mathrm{Ca} \mathrm{I}$ & -0.02 & 0.11 & 3 & -0.06 & 0.12 & 0.16 & & 1 & -0.67 & 0.08 & 5 \\
\hline $\mathrm{Ca}$ II & & & & & & 0.02 & 0.33 & 2 & & & \\
\hline Sc II & -0.07 & 0.18 & 2 & -0.16 & 0.08 & -0.17 & 0.21 & 3 & -0.62 & 0.29 & 3 \\
\hline Ti I & -0.02 & 0.12 & 26 & 0.03 & 0.22 & & & & 0.76 & 0.56 & 9 \\
\hline Ti II & -0.05 & 0.15 & 4 & 0.14 & & -0.54 & 0.13 & 10 & -0.52 & 0.12 & 3 \\
\hline V I & -0.28 & 0.03 & 6 & 0.14 & -0.10 & & & & 0.91 & 0.05 & 2 \\
\hline V II & -0.26 & 0.02 & 2 & -0.09 & & 0.13 & 0.00 & 1 & -0.10 & 0.00 & 1 \\
\hline $\mathrm{Cr} \mathrm{I}$ & -0.12 & 0.14 & 16 & 0.12 & 0.09 & & & & -0.21 & 0.19 & 7 \\
\hline Cr II & 0.23 & 0.14 & 6 & 0.08 & & -0.11 & 0.06 & 13 & -0.04 & 0.16 & 5 \\
\hline Mn I & -0.00 & 0.06 & 5 & 0.06 & - & & & & -0.24 & 0.17 & 4 \\
\hline Mn II & & & & & & 0.24 & 0.03 & 4 & & & \\
\hline $\mathrm{Fe} \mathrm{I}$ & 0.06 & 0.09 & 185 & 0.07 & 0.08 & -0.02 & 0.11 & 10 & -0.02 & 0.08 & 117 \\
\hline $\mathrm{Fe}$ II & 0.05 & 0.07 & 10 & 0.07 & 0.08 & 0.02 & 0.12 & 120 & 0.00 & 0.10 & 26 \\
\hline Co I & -0.10 & 0.10 & 7 & -0.06 & 0.15 & & & & 0.87 & 0.08 & 2 \\
\hline $\mathrm{Ni} \mathrm{I}$ & -0.08 & 0.10 & 43 & 0.11 & 0.00 & & & & 0.17 & 0.09 & 25 \\
\hline Ni II & & & & & & -0.01 & 0.15 & 3 & & & \\
\hline $\mathrm{Cu} \mathrm{I}$ & 0.08 & 0.37 & 5 & 0.15 & 0.21 & & & & 0.30 & 0.26 & 4 \\
\hline $\mathrm{Zn} \mathrm{I}$ & & & & & & & & & 0.72 & 0.11 & 3 \\
\hline Rb I & -0.13 & 0.00 & 1 & - & - & & & & 1.21 & 0.00 & 1 \\
\hline Sr II & & & & & & -0.10 & 0.37 & 2 & & & \\
\hline Y II & 0.09 & 0.08 & 5 & -0.04 & - & & & & 0.53 & 0.10 & 4 \\
\hline $\mathrm{Zr}$ II & -0.04 & 0.14 & 2 & 0.20 & - & & & & 0.47 & 0.18 & 2 \\
\hline La II & -0.05 & 0.14 & 4 & 0.06 & 0.22 & & & & 0.55 & 0.04 & 2 \\
\hline $\mathrm{Ce}$ II & -0.11 & 0.09 & 7 & -0.09 & -0.09 & & & & 0.66 & 0.12 & 6 \\
\hline Pr II & -0.22 & 0.20 & 5 & -0.37 & & & & & 0.72 & 0.28 & 2 \\
\hline $\mathrm{Nd} \mathrm{II}$ & -0.05 & 0.10 & 6 & 0.15 & -0.03 & & & & 0.66 & 0.19 & 6 \\
\hline Sm II & -0.12 & 0.09 & 4 & 0.12 & & & & & 0.62 & 0.03 & 3 \\
\hline Eu II & -0.09 & 0.08 & 3 & 0.18 & 0.04 & & & & 1.44 & 0.93 & 3 \\
\hline Gd II & -0.30 & 0.00 & 1 & - & - & & & & - & - & - \\
\hline
\end{tabular}

\section{Summary}

1. According to the derived RV, color-indices, and parallaxes/distances only 13 of the 20 objects, which we have investigated, are confidently members of Collinder 394.

2. An overwhelming majority of the confident cluster members have high rotational velocity projections, except for star No. 76 (HD 174307) and the Cepheid BB Sgr. Five foreground objects, three K-giants and two MS stars, have low v sinivalues.

3. We have revised color-excesses and reddenings for the objects that allowed us to refine their intrinsic colors, especially for the hot stars.

4. Atmospheric parameters of the hot stars were determined by fitting method, while parameters of the Cepheid, giants, and MS stars were determined exclusively by the method of atmosphere models.

5. The distances of the most cluster stars determined from the GAIA DR2 2018 parallaxes lie in a range of $633-740 \mathrm{pc}$ that roughly corresponds to the mean cluster distance determined earlier by Turner \& Prederos (1985) using photometry. Four MS stars and the Cepheid lie in a range of 760-824 pc, and they could belong to the cluster's corona.
6. Star No. 76 located in the middle of clusters' MS, star No. 61 is located near the "turn-off" point, and HD 174403 is located at the horizontal branch.

7. The abundances of carbon, nitrogen, oxygen, and sodium in the hot star No. 76 turned out to be close to the solar ones, while there is a deficit of $\mathrm{C}$ and an overabundance of $\mathrm{N}$ and $\mathrm{Na}$ with a solar-like $\mathrm{O}$ abundance for the cool variable supergiant BB Sgr. This fact indicates that the Cepheid have already passed through the "first dredge-up" stage.

8. The foreground A-type MS star No. 69 has an anomalous substantial deficit of $\mathrm{CNO}$ elements with a deficit of some $\alpha$ - and an overabundance of $\mathrm{r}$ - and s-process elements.

9. The foreground object an F7-8 MS star No. 75 is a lithium-rich object.

\section{References}

Bessell M.S., Castelli F. \& Plez B.: 1998, A $\& A$, 333, 231.

Castelli F. \& Kurucz R.L.: 2004, arXiv: astro-ph/ $040508 \%$.

Coelho P.R.T.: 2014, MNRAS, 440, 1027.

Conrad C. et al.: 2017, A\&A, 600, 106.

GAIA DR2 2018, CDS/ADS Collection of Electronic Catalogues, 1345, 0. 
Table 5: Chemical abundances for Collinder 394 objects. Part 2.

\begin{tabular}{|c|c|c|c|c|c|c|c|c|c|c|c|c|}
\hline & \multicolumn{3}{|c|}{$21\left(\mathrm{CPD}-20^{\circ} 7255\right)$} & \multicolumn{3}{|c|}{72 (GSC 06289-03067) } & \multicolumn{3}{|c|}{74 (HD 174402) } & \multicolumn{3}{|c|}{$75\left(\mathrm{CPD}-20^{\circ} 7218\right)$} \\
\hline Element & {$[\mathrm{El} / \mathrm{H}]$} & $\sigma$ & $\mathrm{NL}$ & {$[\mathrm{El} / \mathrm{H}]$} & $\sigma$ & NL & {$[\mathrm{E} 1 / \mathrm{H}]$} & $\sigma$ & $\mathrm{NL}$ & {$[\mathrm{El} / \mathrm{H}]$} & $\sigma$ & $\mathrm{NL}$ \\
\hline $\mathrm{C} \mathrm{I}$ & -0.28 & 0.01 & 3 & -0.10 & 0.18 & 4 & 0.02 & 0.05 & 2 & -0.15 & 0.10 & 3 \\
\hline $\mathrm{N} \mathrm{I}$ & -0.13 & 0.00 & 1 & & & - & - & - & - & -0.02 & 0.12 & 2 \\
\hline O I & -0.07 & 0.20 & 3 & -0.07 & 0.13 & 3 & 0.03 & 0.00 & 2 & -0.02 & 0.01 & 2 \\
\hline $\mathrm{Na} \mathrm{I}$ & -0.05 & 0.13 & 2 & 0.23 & 0.03 & 2 & -0.12 & 0.15 & 3 & -0.09 & 0.08 & 2 \\
\hline $\mathrm{Mg} \mathrm{I}$ & 0.09 & 0.15 & 2 & -0.05 & 0.00 & 1 & 0.14 & 0.09 & 2 & -0.05 & 0.00 & 1 \\
\hline $\mathrm{Al} \mathrm{I}$ & 0.06 & 0.08 & 5 & 0.31 & 0.12 & 6 & 0.16 & 0.11 & 6 & 0.01 & 0.08 & 5 \\
\hline $\mathrm{Si} \mathrm{I}$ & 0.13 & 0.10 & 15 & 0.28 & 0.10 & 14 & 0.24 & 0.09 & 10 & 0.02 & 0.13 & 12 \\
\hline Si II & 0.41 & 0.09 & 2 & & & - & & & - & 0.25 & 0.06 & 2 \\
\hline S I & 0.09 & 0.06 & 2 & 0.26 & 0.00 & 1 & 0.46 & 0.00 & 1 & 0.16 & 0.10 & 2 \\
\hline $\mathrm{K}$ I & 0.21 & 0.00 & 1 & 0.11 & 0.00 & 1 & 0.10 & 0.09 & 2 & 0.47 & 0.00 & 1 \\
\hline $\mathrm{Ca} \mathrm{I}$ & -0.01 & 0.14 & 8 & -0.13 & 0.14 & 6 & -0.09 & 0.07 & 7 & -0.01 & 0.18 & 5 \\
\hline Sc II & 0.10 & 0.08 & 5 & 0.12 & 0.08 & 5 & 0.47 & 0.08 & 5 & 0.89 & 0.00 & 1 \\
\hline $\mathrm{Ti} \mathrm{I}$ & 0.04 & 0.21 & 23 & 0.21 & 0.24 & 25 & 0.07 & 0.33 & 23 & -0.01 & 0.11 & 4 \\
\hline Ti II & 0.19 & 0.17 & 5 & 0.18 & 0.30 & 5 & 0.22 & 0.21 & 2 & -0.25 & 0.06 & 3 \\
\hline V I & 0.04 & 0.15 & 6 & 0.07 & 0.17 & 5 & 0.16 & 0.05 & 3 & 0.52 & 0.00 & 1 \\
\hline V II & 0.07 & 0.11 & 3 & 0.27 & 0.13 & 4 & 0.31 & 0.10 & 3 & 0.47 & 0.00 & 1 \\
\hline Cr I & -0.15 & 0.08 & 17 & -0.11 & 0.09 & 16 & -0.20 & 0.09 & 18 & -0.12 & 0.21 & 8 \\
\hline Cr II & 0.05 & 0.05 & 6 & & & & & & & 0.18 & 0.19 & 5 \\
\hline $\mathrm{Mn} \mathrm{I}$ & 0.49 & 0.10 & 5 & 0.66 & 0.23 & 3 & 0.24 & 0.12 & 2 & -0.10 & 0.13 & 6 \\
\hline $\mathrm{Fe} \mathrm{I}$ & 0.12 & 0.12 & 232 & 0.16 & 0.14 & 212 & 0.12 & 0.12 & 190 & 0.06 & 0.15 & 172 \\
\hline $\mathrm{Fe}$ II & 0.10 & 0.10 & 25 & 0.17 & 0.12 & 15 & 0.11 & 0.09 & 2 & 0.07 & 0.14 & 13 \\
\hline Co I & 0.02 & 0.06 & 6 & 0.35 & 0.15 & 8 & 0.24 & 0.19 & 5 & 0.20 & 0.06 & 2 \\
\hline $\mathrm{NiI}$ & 0.03 & 0.06 & 43 & 0.18 & 0.12 & 47 & 0.13 & 0.10 & 43 & -0.05 & 0.17 & 31 \\
\hline $\mathrm{Cu} \mathrm{I}$ & & & . & & 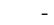 & - & - & - & - & 0.04 & 0.37 & 5 \\
\hline $\mathrm{Zn} \mathrm{I}$ & & & & & & - & & & & 0.01 & 0.07 & 3 \\
\hline $\mathrm{Rb} \mathrm{I}$ & -0.30 & 0.00 & 1 & -0.10 & 0.00 & 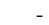 & -0.01 & 0.00 & 1 & & & 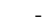 \\
\hline Y II & -0.00 & 0.20 & 3 & 0.03 & 0.21 & 3 & 0.02 & 0.21 & 3 & 0.10 & 0.12 & 3 \\
\hline $\mathrm{Zr} \mathrm{II}$ & -0.05 & 0.07 & 2 & 0.01 & 0.02 & 2 & 0.26 & 0.20 & 3 & 0.51 & 0.00 & 1 \\
\hline La II & 0.10 & 0.05 & 4 & 0.22 & 0.19 & 6 & 0.20 & 0.14 & 4 & 0.05 & 0.00 & 1 \\
\hline Ce II & -0.09 & 0.09 & 3 & -0.01 & 0.07 & 3 & 0.09 & 0.11 & 7 & -0.20 & 0.35 & 2 \\
\hline Pr II & -0.01 & 0.19 & 4 & 0.04 & 0.20 & 4 & 0.10 & 0.24 & 4 & 1.03 & 0.00 & 1 \\
\hline Nd II & 0.29 & 0.07 & 6 & 0.09 & 0.14 & 5 & 0.30 & 0.18 & 5 & 0.24 & 0.00 & 1 \\
\hline $\mathrm{Sm} \mathrm{II}$ & 0.16 & 0.06 & 3 & 0.19 & 0.13 & 3 & 0.44 & 0.11 & 4 & 1.67 & 0.00 & 1 \\
\hline Eu II & 0.32 & 0.09 & 3 & 0.41 & 0.04 & 2 & 0.58 & 0.10 & 3 & 0.70 & 0.00 & 1 \\
\hline Gd II & 0.13 & 0.00 & 1 & & - & - & 0.26 & 0.00 & 1 & 2.44 & 0.00 & 1 \\
\hline
\end{tabular}

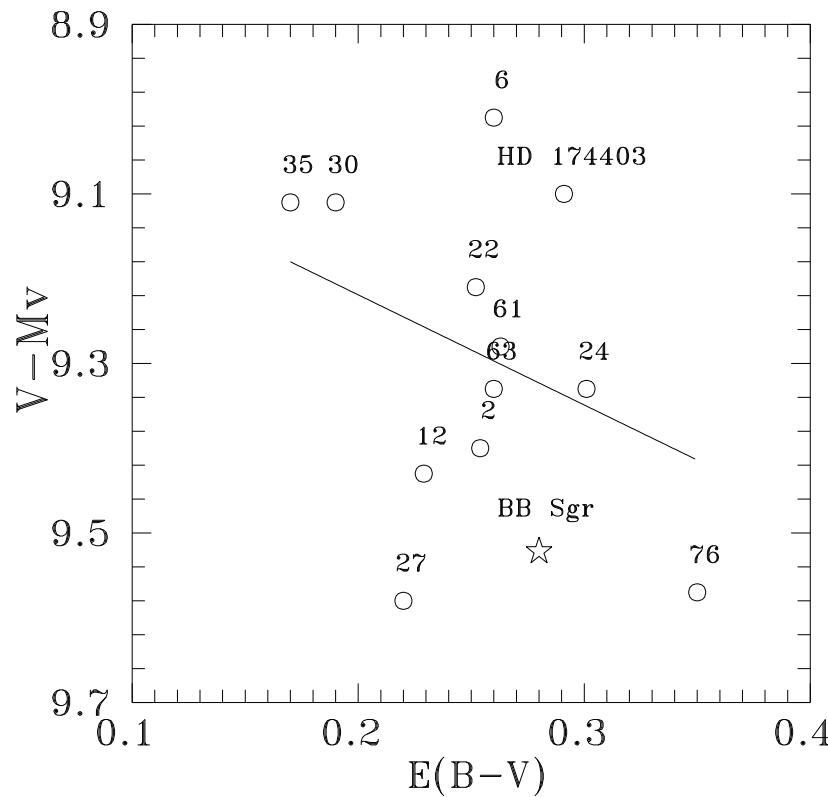

Figure 2: Variable-extinction diagram for confident Collinder 394 members.

Galazutdinov G.A.: 2007, http://gazinur.com/DECHsoftware.html.

Kovtuykh V.V.: 2007, MNRAS, 378, 617.

Kupka F., Piskunov N.E., Ryabchikova T.A., Stempels H.S., Weiss W.W.: 1999, A $6 A, \mathbf{1 3 8}, 119$.

Kurucz R.L.: 1993, CD-ROM No. 23.

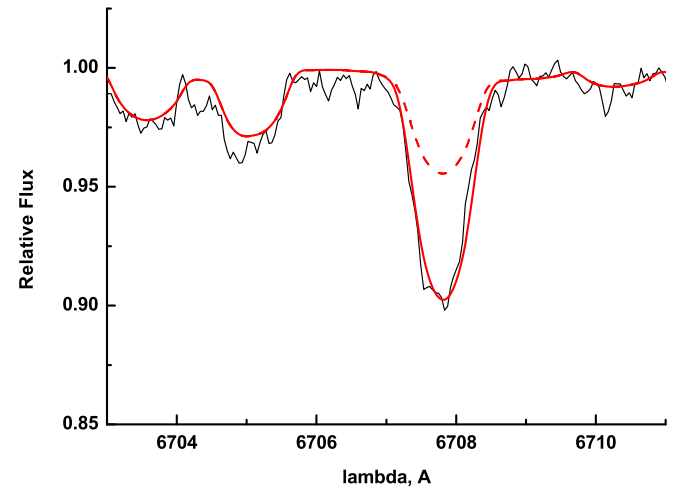

Figure 3: Li I $6707 \AA$ absorption line in star 75 (CPD $-20^{\circ} 7218$ ) atmosphere and its approximation by synth $\left(\mathrm{R}=40000, \mathrm{v} \sin \mathrm{i}=25 \mathrm{~km} \mathrm{~s}^{-1}\right) ; \log \mathrm{A}(\mathrm{Li})=2.5$ (dashed line) and 2.95 (solid line).

Lloyd-Evans, T.: 1968, MNRAS, 141, 109.

Turner D.G.\& Prederos M.: 1983, IBVS, 2263, 1.

Turner D.G.\& Prederos M.: 1985, AJ, 90, 1231.

Usenko I.A., Kniazev A.Yu., Berdnikov L.N., Kravtsov V.V. \& Fokin A.B.: 2013, Astr. Lett., 39, 432.

Wilson R.E. \& Joy A.: 1950, ApJ, 111, 221. 